\title{
Search for Chemically Distinct Nuclei in Galaxies
}

\author{
Olga K. Sil'chenko
}

Sternberg Astronomical Institute, Moscow 119899, Russia

\begin{abstract}
Using the multi-pupil spectrophotometer of the 6-m telescope, five new chemically distinct galactic nuclei are found.
\end{abstract}

\section{Introduction}

Several years ago we (Sil'chenko et al., 1992) announced the discovery of chemically distinct galactic nuclei. Using the first variant of the Multi-Pupil Spectrophotometer (Afanasiev et al., 1990), we have found a jump of Mgb-line equivalent width between the nuclei and their nearest outskirts for 7 galaxies among 12 observed ones. Among those were 3 early-type spirals, 3 S0's, and 1 elliptical.

To extend the field of search for chemically distinct galactic nuclei, I have used a large set of photoelectric multi-aperture data from the catalogue of Longo and Vaucouleurs $(1983,1985)$. By applying an original method of calculation to 350 galaxies with not less than 10 measurements, I have found that about of $25 \%$ elliptical and lenticular galaxies and more than $50 \%$ early-type spiral galaxies have prominently red nuclei (Sil'chenko 1994). A list of galaxies with red nuclei, visible on the northern sky contains 34 objects. These are the primaries which I begin to observe hoping to find new chemically distinct nuclei.

\section{Observations}

Here I present the results of 3D spectroscopy for 5 galaxies. NGC 5533 (Sab) was observed on April 29, 1992. The other 4 galaxies, NGC 1052 (E), 2685 (S0), $6340(\mathrm{Sa})$, and $2841(\mathrm{Sb})$, are taken from the list of 34 galaxies, mentioned above. They have been observed in September - October of 1993. All the observations were made with a new variant of the Multi-Pupil Spectrophotometer of the 6-m telescope. The linear scale was 1.3 arcsec per lens. The common spectral range was 4700-5400 Awith a dispersion of about $2 \AA$ Aer pixel. The equivalent widths of absorption lines $\mathrm{H}_{3}, \mathrm{Mgb}, \mathrm{Fe} I \lambda 5270$ were calculated along the radius. An accuracy of equivalent widths estimated by point-to-point scatter is $0.3 \AA$.

\section{Results}

All the observed galaxies demonstrate a magnesium jump between their nuclei and bulges. But a larger number of points (radius steps), relative to our early work, has allowed to distinguish two different kinds of nucleus decoupling. 


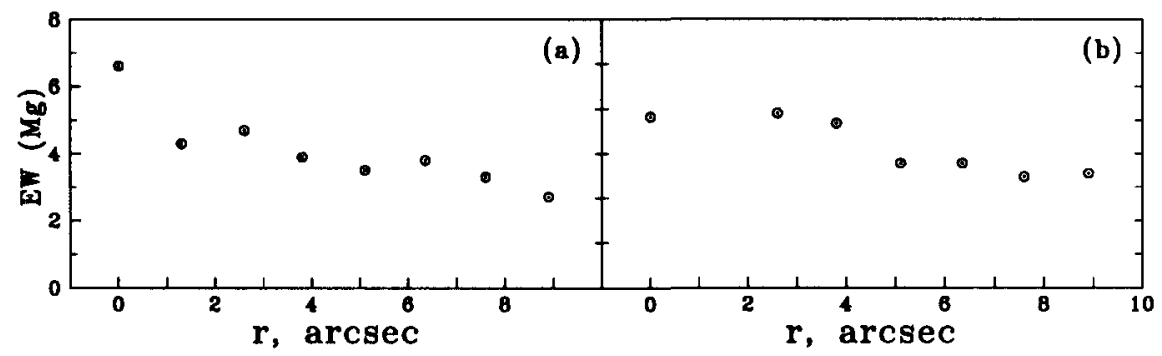

Figure 1. The examples of two kinds of chemically distinct nuclei: NGC 2841 (a) and NGC 1052 (b)

The best example of the first group is NGC 2841 (Fig. 1a). The unresolved nucleus is much more metal-rich than the nearest bulge; but a proper metallicity gradient in the bulge is clearly seen too. If we take only the points belonging to the bulge, we obtain a linear dependence on a radius with a point dispersion of $0.28 \AA$-perfectly according with the observational accuracy of $0.3 \AA$. As the difference between the nuclear $\mathrm{Mgb}$ and the extrapolated bulge linear law is 1.8 $\AA$ - more than $3 \sigma$, - we can state that the nucleus of NGC 2841 is chemically distinct at the confidence level of $99.5 \%$.

Two other disk galaxies, NGC 2685 and 5533, show the Mgb behaviour similar to that of NGC 2841 though slightly less pronounced.

The second group includes NGC 1052 (Fig. 1b) and NGC 6340, which demonstrate RESOLVED chemically distinct nuclei. In both galaxies the jump of magnesium strength is located at $r$ of 4 arcsec. It is not an effect of seeing: the galaxies with unresolved nuclei were observed under the same seeing quality. In NGC 1052 the nucleus has Mgb of $5.07 \pm 0.09$ Aand the bulge has that of $3.52 \pm 0.10 \AA$; in NGC 6340 the nucleus has Mgb of $4.08 \pm 0.11$ Aand the bulge has that of $2.50 \pm 0.25 \AA$. So, in both cases, the difference between the nucleus and the bulge is about of $1.6 \AA$ - more than $3 \sigma$. It is interesting that in NGC 6340 Vigroux et al. (1988) observed a jump of B-R colour from 1.6 to 1.3 at the same radius of 5 arcsec.

\section{References}

Afanasiev, V. L., Vlasiuk, V. V., Dodonov, S. N., and Sil'chenko, O. K. 1990. Preprint SAO RAS No54

Longo, G. and Vaucouleurs, A. 1983. A General Catalogue of Photoelectric Magnitudes and Colors in the UBV System. Austin: Univ. Texas Press

Longo, G. and Vaucouleurs, A. 1985. Supplement to the General Catalogue of Photoelectric Magnitudes and Colors of Galaxies in the UBV System. Austin: Univ. Texas Press

Sil'chenko, O. K., Afanasiev, V. L., and Vlasiuk, V. V. 1992. $A Z h, 69,1121$

Sil'chenko, O. K. 1994. $A Z h$, in press

Vigroux, L., Souviron, J., Lachièze-Rey, M., and Vader, J. P. 1988. $A \in A S$, 73, 